\title{
HMP19 is a new metastasis suppressor in epithelial ovarian cancer
}

\author{
Yuanlin Liu ${ }^{1}$, Shuang Liu², Yingjing Wang ${ }^{2}$, Min Su², Yuquan Zhang ${ }^{1}$, Xiaoqin Liu ${ }^{1}$, \\ Feng Yao, Yunzhao $\mathrm{Xu}^{1 *}$ \\ ${ }^{1}$ Department of Obstetrics and Gynecology, Affiliated Hospital of Nantong University, Nantong, Jiangsu, 226001, China; \\ ${ }^{2}$ Department of Clinical Bio-Bank, Affiliated Hospital of Nantong University, Nantong, Jiangsu, 226001, China
}

\begin{abstract}
HMP19 is a neuron-specific gene; its expression product belongs to a family of neuronal proteins which can be found in numerous kinds of human cancers. However, the clinicopathological significance of HMP19 expression in epithelial ovarian cancer (EOC) is as yet unknown. In this study, protein expression levels of HMP19 in cancerous tissues were determined by tissue microarray immunohistochemistry analysis (TMA-IHC) $(n=117)$. HMP19 protein levels in cancer tissues were associated with clinical characteristics and overall survival rates of patients with EOC. It was found that both mRNA and protein levels of HMP19 were significantly lower in EOC than those in normal ovary or fallopian tube tissues $(P<0.05)$. The protein expression level of HMP19 was significantly associated with a lower FIGO stage, a lower level of CA-125 and a lower presence of metastasis. Consistent with related adverse clinical pathological features, the overall survival (OS) rate of patients with low or non HMP19-expressing tumors was inferior compared to those with high HMP19-expressing tumors. This is in accordance with further studies that found high HMP19 protein level to be an independent prognostic factor for OS in EOC. Multivariate analysis demonstrated that tumor patients with low HMP19 expression had an exceedingly poor OS. HMP19 plays a role in metastasis/tumor suppression and offers a prognostic value for EOC. HMP19, as a new inhibitor, strongly inhibits metastasis and partially attenuates tumor growth in EOC.
\end{abstract}

Keywords: HMP19, human epithelial ovarian cancer, immunohistochemistry, qRT-PCR, prognosis

\section{INTRODUCTION}

The most deadly carcinoma among gynecological malignancies is ovarian cancer ${ }^{[1]}$. It shows no specific signs or symptoms and there are no established screening programs, which frequently leads to late-stage diagnoses ${ }^{[2]}$. The 5 -year survival rate has remained constant at only $39 \%$ over the past 30 years ${ }^{[3]}$. The tumor recurrence is the main cause of ovarian cancer treatment failure following surgery, which seriously

*Correspondence to: Yunzhao Xu, Department of Obstetrics and Gynecology, Affiliated Hospital of Nantong University, Nantong, Jiangsu, 226001, China. E-mail: 455508972@qq.com.

Conflict of interests: The authors declared no conflict of interests.. threatens the life of affected women ${ }^{[4]}$. Its poor prognosis is largely attributed to our poor understanding of the events that initiate ovarian cancer and promote the diseases' progress $^{[5,6]}$.

Prognosis is primarily determined by the presence or absence of metastasis ${ }^{[7]}$. So far, more than 30 metastasis inhibiting genes have been verified as being able to control cancer metastasis ${ }^{[8,9]}$. However, only some anecdotal clinical studies have described the relationship between these genes and the outcomes of ovarian cancer. Therefore, novel biomarkers that have more sensitivity and specificity are urgently needed for early diagnosis and for use as targeted therapies of this disease ${ }^{[10]}$.

$H M P 19$, also known as $N S G 2^{[11]}$, is a neuronspecific gene encoding a $19 \mathrm{kDa}$ protein that is 
located in the Golgi apparatus and belongs to a family of neuronal proteins which includes related family members: neuronal enriched protein NEEP21 (NSG1) and calcyon neuron specific vesicular protein $(\mathrm{CALY})^{[12,13]}$. HMP19 inhibits metastasis and also controls the growth of pancreatic tumors ${ }^{[14]}$. When HMP19 is over-expressed or knocked down, the localization of ERK1/2 activated in the nucleus changed, which promote the cell proliferation ${ }^{[15]}$. After surgery, patients who have a high expression of HMP19 in their pancreatic tumor have a significantly lower incidence of liver metastasis and a better prognosis.

However, little attention has been directed towards the relationship between HMP19 protein expression and clinical parameters in ovarian cancer, and no studies have reported its prognostic value for ovarian cancer. Therefore, in our current study, we aimed to analyze the correlation between patients' overall survival and clinical characteristics, with mRNA and protein expression levels of HMP19 measured via qRT-PCR and tissue microarray immunohistochemistry(TMA-IHC)analysis.

\section{MATERIALS AND METHODS}

\section{Clinical data and tissue specimens of ovarian cancer patients}

In total, 117 tissue samples from patients who underwent surgery at the Gynecology Department of the Affiliated Hospital of Nantong University from 2005 to 2009 were embedded in paraffin and fixed in formalin.

To achieve maximal tumor resection, all ovarian cancer patients were subjected to standardized surgery and platinum-based chemotherapy for $6-8$ cycles after resection. The control group (normal ovarian and fallopian tube samples) was collected from patients who underwent hysterectomy for non-ovarian disease. Before the operation, no patient had been treated with radiation, chemotherapy, or immunotherapy. In the cases of ovarian carcinoma, 83 were serous carcinoma, 18 were endometrioid tumors, and 16 were other types. Among them, 74 patients were in stage I - II and 43 patients were in stage III - IV . At the histological grading, 90 patients had high grade while 27 cases had low grade. All subsequent patient information was updated on July 31, 2017, and the patients were retrospectively studied by consulting the medical records from the Chinese Public Security Bureau. The present study and protocol gained permission from the Human Research Ethics Committee of the Affiliated Hospital of Nantong University, Jiangsu province,
China, and all experiments were performed in accordance with the Affiliated Hospital of Nantong University's approved guidelines.

\section{Immunohistochemistry (IHC)}

TMA section was deparaffinized, and then incubated in $3 \% \mathrm{H}_{2} \mathrm{O}_{2}$. Endogenous peroxidase underwent decomposition with methanol for $15 \mathrm{~min}$. The sections were heated in sodium citrate to buffer the antigen retrieval $(10 \mathrm{mmol} / \mathrm{L}, \mathrm{pH} 6.0)$ for $3 \mathrm{~min}$ in a pressure cooker. In the second step, the tissue sections were incubated with the primary goat antiHMP19 antibody (Abcam, Cambridge, UK) diluted 1:200 in $1 \%$ bovine serum albumin for $1 \mathrm{~h}$. Then the sections were washed with phosphate-buffered saline, and horseradish peroxidase-conjugated donkey anti-goat antibody (Abcam, Cambridge, UK) was incubated in tissue sections, and washed again after $15 \mathrm{~min}$. The color was cultivated by incubating with diaminobenzidine solution (Kem-En-Tec Diagnostics, Denmark) then by applying light counterstaining with hematoxylin.

The Olympus BX53 microscope (Olympus Co., Tokyo, Japan) was used to observe the quantification of HMP19 immunostaining. Two investigators were blinded to the sample identities. The staining intensity was scored as follows: 0 (-, no staining), $1(+$, mild staining), $2(++$, medium staining), or $3(+++$, intense staining). The percentage of cells was also scored. The final HMP19 staining score was considered as the intensity and percentage scores.

Then, the cut-off point was chosen because it had a significant meaning in view of overall survival (OS) by employing the software package X-tile (The Rimm Lab, Yale University; http:// www.tissuearray. org/rimmlab) ${ }^{[15,16]}$. In our study, the selected HMP19 protein expression cut-off point was 100 ; a score below 100 was defined as low expression, while a score between 100 and 300 was defined as high ex$\operatorname{pression}^{[17]}$.

\section{Statistical analysis}

Chi-square test was conducted to determine whether HMP19 protein expression was related to clinicopathological parameters. Then, using univariate and multivariate Cox regression models to determine the prognostic factors. The Kaplan-Meier method was used to calculate survival curves of the patients. In all analyses, $P<0.05$ was considered as statistically significant. The data were analyzed using STATA 12.0 (StataCorp, College Station,TX, USA) and SPSS 20 statistical software (SPSS Inc., Chicago, IL, USA). 


\section{RESULTS}

\section{HMP19 protein expression patterns by IHC in tissue arrays of EOC patients}

TMA-based immunohistochemistry was investigated to determine HMP19 protein expression in archived ovarian tissue blocks at the tissue level, including 117 EOC samples, 30 cases of benign ovarian tumors, 24 samples of normal ovarian samples, 20 borderline ovarian tumor tissues, and 20 normal fallopian tube samples.

HMP19 protein expression was lower in EOC samples and significantly superior in borderline ovarian tumor samples and normal fallopian tube samples. Low HMP19 expression was observed in $55.56 \%$ of ovarian cancer tissues; however, $75.00 \%-91.67 \%$ of normal or benign ovarian samples had detectable ex- pression (Table 1, Fig.1). HMP19 expression in noncancerous tissues was significantly superior to that in EOC tissues (Pearson $\chi^{2}=19.442, P=0.001$ ).

Table 1 Immunohistochemical staining of HMP19 protein in normal ovarian, normal fallopian tube, benign ovarian tumor, borderline ovarian tumor and EOC tissues

\begin{tabular}{lccccc}
\hline \multirow{2}{*}{ Tissue sample } & $n$ & \multicolumn{4}{c}{ HM19 expression } \\
\cline { 3 - 6 } & & $\begin{array}{c}\text { Low or } \\
\text { none }\end{array}$ & High & Pearson $\chi^{2}$ & $P$-value \\
\hline Normal ovarian tissue & 24 & $22(91.67)$ & $2(8.33)$ & 19.442 & 0.001 \\
Normal fallopian tube & 20 & $15(75.00)$ & $5(25.00)$ & \\
tissue & 30 & $25(83.33)$ & $5(16.67)$ & \\
Benign ovarian tumor & & & & \\
Borderline ovarian & 20 & $16(80.00)$ & $4(20.00)$ & \\
tumor & 119 & $65(55.56)$ & $52(44.44)$ & \\
EOC & & & &
\end{tabular}

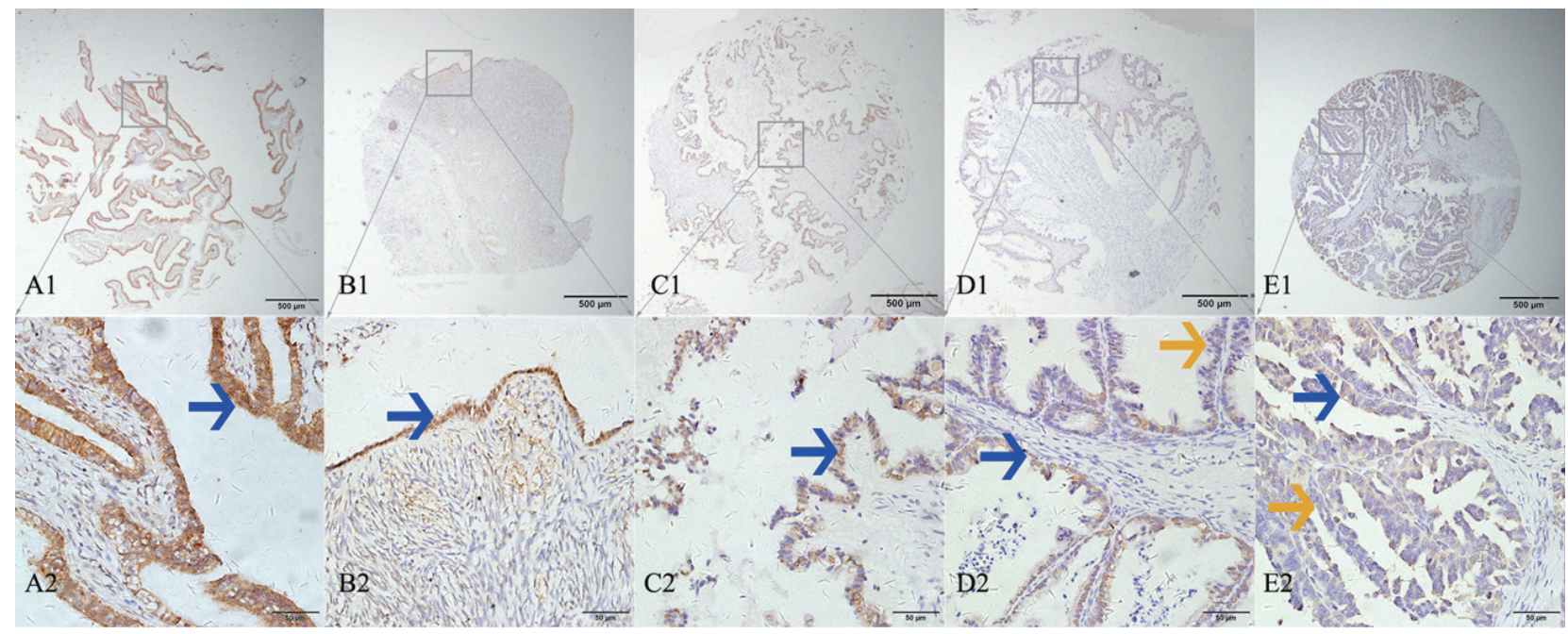

Fig.1 HMp19 protein was determined in ovarian cancer tissue, borderline ovarian tumor samples, normal ovarian tissue and normal fallopian tube tissue by TMA-IHC. (A1-A2) Strong positive for HMP19 expression of normal fallopian tube tissue. (B1-B2) Strong positive for HMP19 expression of normal ovarian tissue. (C1-C2) Weakly positive for HMP19 expression of benign ovarian tumor samples. (D1-D2) Weakly positive for HMP19 expression of borderline ovarian tumor samples. (E1-E2) Negative for HMP19 expression of ovarian serous papillary carcinoma. Original magnification $\times 40(\mathrm{bar}=500 \mu \mathrm{m})$ in $(\mathrm{Al}, \mathrm{B} 1, \mathrm{Cl}, \mathrm{D} 1, \mathrm{E} 1) ; \times 400(\mathrm{bar}=50 \mu \mathrm{m})$ in $(\mathrm{A} 2, \mathrm{~B} 2, \mathrm{C} 2, \mathrm{D} 2, \mathrm{E} 2)$.

\section{Association of HMP19 expression with EOC clinical characteristics}

The correlation between HMP19 and ovarian cancer patients' clinical features were evaluated and the results are summarized in Table 2 . It was found that low HMP19 expression was significantly associated with higher stage (FIGO stage III - IV,$P<0.001$ ), presence of metastasis $(P=0.024)$, and level of CA$125(P<0.001)$.

\section{Low HMP19 protein expression predicts poor overall survival}

Using univariate and multivariate analyses to identify prognostic factors for EOC, which was performed using Cox regression models for all significant variables. In univariate analysis, the following markers are correlated with poor overall survival: low HMP19 expression (HR=0.268, $P<0.001,95 \% \mathrm{CI}$ : $0.133-0.540$ ), older age at diagnosis $(\mathrm{HR}=2.121, P=0.010,95 \% \mathrm{CI}$ : 1.195-3.763;), higher tumor grade (HR=3.166, $P=$ 0.015, 95\%CI: 1.251-8.010), positive lymph nodes (HR=3.267, $P<0.001,95 \%$ CI: 1.721-6.201), presence of metastasis $(\mathrm{HR}=6.015, P<0.001,95 \% \mathrm{CI}$ : $3.210-$ 11.269), and higher FIGO stage (HR: 4.893, $P<0.001$, 95\%CI: 2.696-8.880). Because lymph node positivity and metastasis were included in FIGO stage, they were excluded from the multivariate analysis. In the multivariate analysis, lower HMP19 expression $(\mathrm{HR}=0.453$, 
Table 2 Correlation of HMP19 protein expression with EOC patients' clinicopathologic characteristics

\begin{tabular}{|c|c|c|c|c|c|}
\hline \multirow{2}{*}{ Groups } & \multirow{2}{*}{$n$} & \multicolumn{4}{|c|}{$\operatorname{HMP} 19[n(\%)]$} \\
\hline & & Low & High & Pearson $\chi^{2}$ & $P$-value \\
\hline Total & 117 & $65(55.56)$ & $52(44.44)$ & & \\
\hline Age & & & & 0.357 & 0.571 \\
\hline$\leqslant 60$ years & 73 & $39(53.42)$ & $34(46.58)$ & & \\
\hline$>60$ years & 44 & $26(59.09)$ & 18(40.91) & & \\
\hline FIGO stage & & & & 9.797 & $0.002^{*}$ \\
\hline $\mathrm{I} \sim \mathbb{I}$ & 74 & $33(44.59)$ & $41(55.41)$ & & \\
\hline III $\sim$ IV & 43 & $32(74.42)$ & $11(25.58)$ & & \\
\hline Grade & & & & 0.195 & 0.666 \\
\hline Low grade & 27 & 14(51.85) & 13(48.15) & & \\
\hline High grade & 90 & $51(56.67)$ & $39(43.33)$ & & \\
\hline $\begin{array}{l}\text { Histological clas- } \\
\text { sification }\end{array}$ & & & & 0.599 & 0.741 \\
\hline Serous carcinoma & 83 & $48(57.83)$ & $35(42.17)$ & & \\
\hline $\begin{array}{l}\text { Endometrioid } \\
\text { carcinoma }\end{array}$ & 18 & $9(50.00)$ & $9(50.00)$ & & \\
\hline Other $^{\mathrm{a}}$ & 16 & $8(50.00)$ & $8(50.00)$ & & \\
\hline Lymph nodes & & & & 0.675 & 0.463 \\
\hline Yes & 91 & $50(54.95)$ & $41(45.05)$ & & \\
\hline No & 20 & $13(65.00)$ & $7(35.00)$ & & \\
\hline Metastasis & & & & 5.265 & $0.024^{*}$ \\
\hline Yes & 72 & $34(47.22)$ & $38(52.78)$ & & \\
\hline No & 45 & $31(68.89)$ & 14(31.11) & & \\
\hline Ascites & & & & 0.322 & 0.680 \\
\hline Yes & 59 & $32(54.24)$ & $27(45.76)$ & & \\
\hline No & 40 & $24(60.00)$ & $16(40.00)$ & & \\
\hline Ascites cell & & & & 0.734 & 0.477 \\
\hline Yes & 63 & $34(53.97)$ & $29(46.03)$ & & \\
\hline No & 25 & $16(64.00)$ & $9(36.00)$ & & \\
\hline CA199 & & & & 0.010 & 1.000 \\
\hline Yes & 73 & $46(63.01)$ & $27(36.99)$ & & \\
\hline No & 13 & $8(63.01)$ & $5(38.46)$ & & \\
\hline CA125 & & & & 18.555 & $0.001^{*}$ \\
\hline$\leqslant 100$ & 18 & $4(22.22)$ & $14(77.78)$ & & \\
\hline$>100$ & 65 & $50(76.92)$ & $15(23.08)$ & & \\
\hline CA153 & & & & 2.457 & 0.148 \\
\hline Yes & 28 & $14(50.00)$ & $14(50.00)$ & & \\
\hline No & 30 & $34(68.00)$ & $16(32.00)$ & & \\
\hline
\end{tabular}

${ }^{*} P<0.05$ indicates a significant association among the variables; Metastasis: pelvic lymph node metastases or nearby tissues and organs involved. a, others: clear cell carcinoma, 5 cases; mucinous carcinoma, 6 cases; transitional cell carcinoma, 3 cases; adeno-squamous carcinoma, 3 cases.

fallopian tube and normal ovarian tissues was significantly higher than that in ovarian cancer at the protein level. Lower HMP19 protein level was related to FIGO stages, metastasis and a higher serum CA125 level. These results suggest that HMP19 can be used as a new biomarker for ovarian carcinoma. As a result, low HMP19 is an independent prognostic factor for poor overall survival in EOC. In addition, it is possible that HMP19 may be a target for anti-tumor therapy, and its study may clarify the link between ovarian cancer and tumor development.

However, our study also has several limitations. First of all, because it is a retrospective study, it is affected by sample selection bias, owing to this future
$P=0.034$, 95\% CI: 0.218-0.944), and higher FIGO stage $(\mathrm{HR}=2.207, P=0.030,95 \% \mathrm{CI}$ : $1.708-4.57)$ were independent factors correlated with survival (Table 3). Kaplan-Meier survival curves were used to show similar results (log rank, $P<0.001$, Fig.2).

\section{DISCUSSION}

In ovarian cancer, immunotherapy is a promising way to improve therapeutic effectiveness and survival. The neuron-specific gene family $(N S G)^{[18]}$, encoded a family of endocytic proteins ${ }^{[19]}$, plays an important role in regulating excitatory synaptic transmission. Kurahara et al. found that in pancreatic cancer, HMP19 was a metastasis suppressor, altering signaling leading to cell proliferation, tumor size, plexus invasion, and liver metastasis, and seemed to have prognostic value ${ }^{[14]}$. As far as we know, no research has been previously reported on the correlation between HMP19 and the survival of patients with EOC.

It is worth noting that little has been previously reported about HMP19. In neural and neuroendocrine cells ${ }^{[20]}, H M P 19$ encodes a $19 \mathrm{kDa}$ protein that localizes in the Golgi apparatus. In Huntington's disease, malfunction of HMP19 has been implicated as part of the pathogenesis ${ }^{[17,21]}$. In pancreatic cancer, Kurahara et al. found that HMP19 may have aberrant activity as a downstream effector through the ERK1/2 pathways, and in their in vitro and in vivo experiments, HMP19 expression significantly inhibited tumor growth ${ }^{[14]}$. ERK1/2 signaling controls a wide diversity of cellular functions, such as survival, proliferation, migration, and chemotherapeutic drug resistance ${ }^{[22]}$. Therefore, the ERK1/2 pathway is considered to be a crucial target for therapeutic intervention ${ }^{[23,24]}$. In the future, we plan to explore potential relationships between HMP19 and ERK1/2 pathways further.

HMP19 is frequently expressed in other types of cancers, and can provide additional prognostic information in pancreatic ductal adenocarcinoma (PDAC) and leukemia ${ }^{[25,26]}$. HMP19 intensely suppresses the growth and metastasis of PDAC tumors. In addition, high expression of HMP19 protein is strongly correlated with longer survival and lower rates of liver metastasis in patients with PDAC ${ }^{[14]}$.

So far, however, there has been no published research examining any potential correlation of HMP19 with the survival of patients suffering ovarian cancer. This report suggests for the first time that HMP19 may be used as a novel factor to predict the clinical outcomes of patients with ovarian cancer.

In our study, the expression of HMP19 in normal 
Table 3 Prognostic markers for overall survival in EOC patients by univariate and multivariate Cox proportional hazard model analysis

\begin{tabular}{|c|c|c|c|c|c|c|}
\hline \multirow{2}{*}{ Variable } & \multicolumn{3}{|c|}{ Univariate analysis } & \multicolumn{3}{|c|}{ Multivariate analysis } \\
\hline & HR & $p$ value & $95 \% \mathrm{CI}$ & HR & $p$ value & $95 \% \mathrm{CI}$ \\
\hline HMP19 expression: Low versus High & 0.268 & 0.001 & $0.133-0.540$ & 0.453 & 0.034 & $0.218-0.944$ \\
\hline Age (years): $\leqslant 60$ versus $>60$ & 2.121 & 0.010 & $1.195-3.763$ & & & \\
\hline Grade: Low versus high & 3.166 & 0.015 & $1.251-8.010$ & & & \\
\hline Single or double: None versus yes & 1.900 & 0.029 & $1.067-3.384$ & 0.454 & 0.028 & $0.224-0.918$ \\
\hline Type: Serous versus others & 0.542 & 0.877 & $0.576-1.337$ & & & \\
\hline Metastasis: None versus yes & 6.015 & 0.001 & $3.210-11.269$ & 5.145 & 0.001 & $2.409-10.985$ \\
\hline Ascites: None versus yes & 1.048 & 0.878 & $0.574-1.914$ & & & \\
\hline Ascites cell: None versus yes & 1.855 & 0.063 & $0.967-3.558$ & & & \\
\hline FIGO: Stage I versus stage $\Pi$ - IV & 4.893 & 0.001 & $2.696-8.880$ & 2.207 & 0.030 & $1.708-4.570$ \\
\hline
\end{tabular}
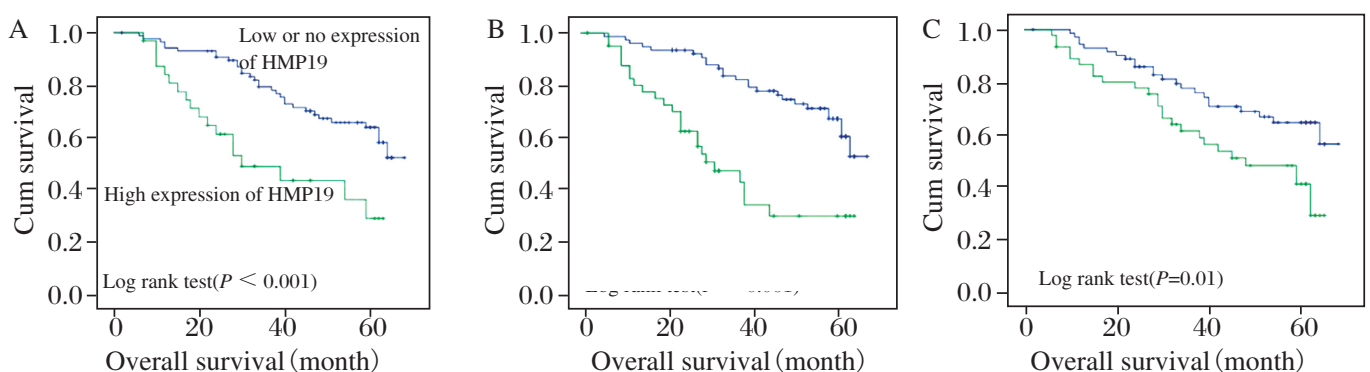

Fig.2 Survival curves of EOC patients by the Kaplan-Meier method and the log-rank test. (A) HMP19 EOC patients(green line, 1) had significantly better overall survival than HMP19- patients (blue line, 0); (B) EOC patients with advanced stage (FIGO III - IV stage) (green line, 1) had significantly worse overall survival than patients with early stage (FIGO I - II stage) (blue line, 0); (C) EOC patients diagnosed at older age ( $>60 y e a r s)$ (green line, 1) had significantly worse overall survival than patients diagnosed at younger age ( $\leqslant 60$ years) (blue line, 0 ).

studies are needed to confirm the results, including additional EOC cases. Furthermore, we did not investigate the mechanism of HMP19 in ovarian cancer. Further studies are needed to explore the role of HMP19 and whether its ligands can be identified as a potential therapeutic target of EOC.

In conclusion, our study has demonstrated critical insight into HMP19 and shown it is to be an independent prognostic marker of ovarian carcinoma. These findings suggest that HMP19 is a profound inhibitor of EOC metastasis. Further investigation of the mechanisms by which HMP19 suppresses tumor growth may disclose a new therapeutic target for patients of ovarian cancer. As a new biomarker, the potential clinical value of HMP19 should be studied in a randomized controlled trial.

\section{Acknowledgments and fundings}

This study was supported by Grants from the Frontier and Key Technologies Research Projects (NO.MS22015093) and the Nantong $\mathrm{Mu}-$ nicipal Science and Technology Project (NO. MS12015006). We thank Wendy Bruening PhD from Liwen Bianji, Edanz Group China (www.liwenbianji.cn/ac) for editing the English text of a draft of this manuscript.

\section{References}

[1] Worzfeld T, Pogge Von Strandmann E, Huber M, et al. The unique molecular and cellular microenvironment of ovarian cancer. Front Oncol, 2017, 7:24.

[2] Kim JH, Park CW, Um D, et al. Mass spectrometric screening of ovarian cancer with serum glycans. Dis Markers, 2014, 2014: 634289.

[3] Corney DC, Hwang CI, Matoso A, et al. Frequent downregulation of miR-34 family in human ovarian cancers. Clin Cancer Res, 2010, 16(4): 1119-28.

[4] Pal SK, Figlin RA, Reckamp K. Targeted therapies for non-small cell lung cancer: an evolving landscape. Mol Cancer Ther, 2010, 9(7): 1931-44.

[5] Parkinson DR, Johnson BE, Sledge GW. Making personalized cancer medicine a reality: challenges and opportunities in the development of biomarkers and companion diagnostics. Clin Cancer Res, 2012, 18(3): 61924.

[6] Xu Y, Wang C, Zhang Y, et al. Overexpression of MAGE-a9 is predictive of pooor prognosis in epithelial ovarian cancer. Sci Rep, 2015, 5:12104.

[7] Alonso DF, Ripoll GV, Garona J, et al. Metastasis: recent discoveries and novel perioperative treatment strategies with particular interest in the hemostatic compound desmopressin. Curr Pharm Biotechnol, 2011, 12(11): 1974-80.

[8] Liu W, Vivian CJ, Brinker AE, et al. Microenvironmental Influences on metastasis suppressor expression and 
function during a metastatic cell's Journey. Cancer Microenviron, 2014, 7(3): 117-31.

[9] Bohl CR, Harihar S, Denning WL, et al. Metastasis suppressors in breast cancers: mechanistic insights and clinical potential. J Mol Med (Berl), 2014, 92(1): 13-30.

[10] Kelly K, Huang C. Biological agents in non-small cell lung cancer: a review of recent advances and clinical results with a focus on epidermal growth factor receptor and vascular endothelial growth factor. J Thorac Oncol, 2008, 3(6): 664-73.

[11] Goehler H, Lalowski M, Stelzl U, et al. A protein interaction network links GIT1, an enhancer of huntingtin aggregation, to Huntington's disease. Mol Cell, 2004, 15(6): 853-65.

[12] Digilio L, Yap CC, Winckler B. Ctip2-, Satb2-, Prox1-, and GAD65-Expressing neurons in rat cultures: Preponderance of single- and double-positive cells, and cell type-specific expression of neuron-specific gene family members, Nsg-1 (NEEP21) and Nsg-2 (P19). PLoS One, 2015, 10(10): e0140010.

[13] Muthusamy N, Ahmed SA, rana BK, et al. Phylogenetic analysis of the NEEP21/calcyon/P19 family of endocytic proteins: evidence for functional evolution in the vertebrate CNS. J Mol Evol, 2009, 69(4): 319-32.

[14] Kurahara H, Bohl C, Natsugoe S, et al. Suppression of pancreatic cancer growth and metastasis by HMP19 identified through genome-wide shRNA screen. Int $J$ Cancer, 2016, 139(3): 628-38.

[15] Dong QZ, Wang Y, Tang ZP, et al. Derlin-1 is overexpressed in non-small cell lung cancer and promotes cancer cell invasion via EGFR-ERK-mediated upregulation of MMP-2 and MMP-9. Am J Pathol, 2013, 182(3): 954-64.

[16] Lu C, Wang X, Zhu H, et al. Over-expression of ROR2 and Wnt5a cooperatively correlates with unfavorable prognosis in patients with non-small cell lung cancer. Oncotarget, 2015, 6(28): 24912-21.

[17] Sun J, Nan G. The extracellular signal-regulated ki- nase 1/2 pathway in neurological diseases: A potential therapeutic target (Review). Int J Mol Med, 2017, 39(6): $1338-46$.

[18] Barford K, Yap CC, Dwyer ND, et al. The related neuronal endosomal proteins NEEP21 (Nsg1) and P19 (Nsg2) have divergent expression profiles in vivo. J Comp Neurol, 2017, 525(8): 1861-78.

[19] Davidson HT, Xiao J, Dai R, et al. Calcyon is necessary for activity-dependent AMPA receptor internalization and LTD in CAl neurons of hippocampus. Eur J Neurosci, 2009, 29(1): 42-54.

[20] Ueda T, Nagamachi A, Takubo K, et al. Fbxll0 overexpression in murine hematopoietic stem cells induces leukemia involving metabolic activation and upregulation of Nsg2. Blood, 2015, 125(22): 3437-46.

[21] Ishii A, Furusho M, Dupree JL, et al. Strength of ERK1/2 MAPK activation determines its effect on myelin and axonal integrity in the adult CNS. J Neurosci, 2016, 36(24): 6471-87.

[22] Balmanno K, Cook SJ. Tumour cell survival signalling by the ERK1/2 pathway. Cell Death Differ, 2009, 16(3): 368-77.

[23] Lefloch R, Pouyssegur J, Lenormand P. Single and combined silencing of ERK1 and ERK2 reveals their positive contribution to growth signaling depending on their expression levels. Mol Cell Biol, 2008, 28(1): 511-27.

[24] Lake D, Correa SA, Muller J. Negative feedback regulation of the ERK1/2 MAPK pathway. Cell Mol Life Sci, 2016, 73(23): 4397-413.

[25] Cancer Genome Atlas Research N et work, Ley TJ, miller c, et al. Genomic and epigenomic landscapes of adult de novo acute myeloid leukemia. N Engl J Med, 2013, 368(22): 2059-74.

[26] Wu X, Johansen JV, Helin K. Fbx110/Kdm2b recruits polycomb repressive complex 1 to $\mathrm{CpG}$ islands and regulates H2A ubiquitylation. Mol Cell, 2013, 49(6): 1134-46.

(Received 28 May 2018, Revised 16 July 2018, Accepted 13 August 2018) 